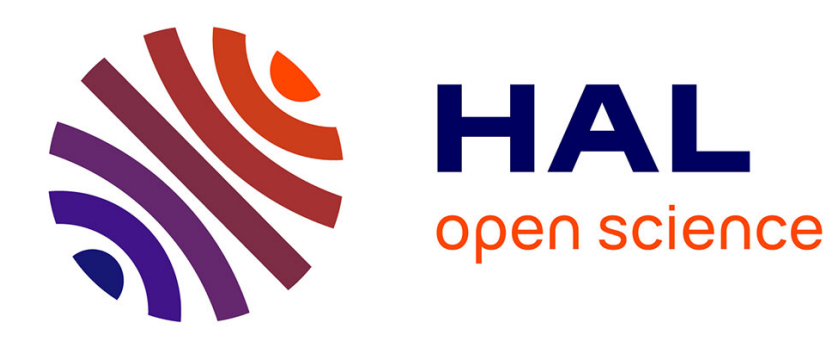

\title{
On alternative theorems and necessary conditions for efficiency
}

Do Van Luu, Manh-Hung Nguyen

\section{To cite this version:}

Do Van Luu, Manh-Hung Nguyen. On alternative theorems and necessary conditions for efficiency. 2006. halshs-00112454

\section{HAL Id: halshs-00112454 \\ https://shs.hal.science/halshs-00112454}

Submitted on 8 Nov 2006

HAL is a multi-disciplinary open access archive for the deposit and dissemination of scientific research documents, whether they are published or not. The documents may come from teaching and research institutions in France or abroad, or from public or private research centers.
L'archive ouverte pluridisciplinaire $\mathbf{H A L}$, est destinée au dépôt et à la diffusion de documents scientifiques de niveau recherche, publiés ou non, émanant des établissements d'enseignement et de recherche français ou étrangers, des laboratoires publics ou privés. 


\title{
Centre d'Economie de la Sorbonne
}

UMR 8174

\author{
On alternative theorems and \\ necessary conditions for efficiency \\ Do Van LUU
}

Manh Hung NGUYEN

2006.19
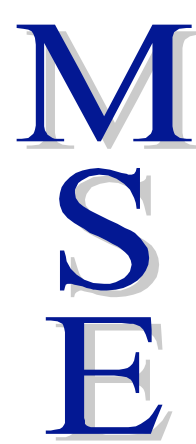

la

de

CENTRE NATIONAL

DE LA RECHERCHE

SCENTIFIQUE

Maison des Sciences Économiques, 106-112 boulevard de L'Hôpital, 75647 Paris Cedex 13

http://mse.univ-paris1.fr/Publicat.htm 


\title{
ON ALTERNATIVE THEOREMS AND NECESSARY CONDITIONS FOR EFFICIENCY
}

\author{
Do Van Luu and Manh Hung Nguyen
}

\begin{abstract}
In this paper, we establish theorems of the alternative for a system described by inequalities, equalities and an inclusion, which are generalizations of Tucker's classical theorem of the alternative, and develop KuhnTucker necessary conditions for efficiency to mathematical programs in normed spaces involving inequality, equality and set constraints with positive Lagrange multipliers of all the components of objective functions.
\end{abstract}

\section{INTRODUCTION}

Theorems of the alternative play an important role in establishing necessary optimality conditions to scalar and multiobjective optimization problems. Under suitable constraint qualifications we could obtain Kuhn-Tucker necessary conditions for efficiency in terms of derivatives or directional derivatives of objective and constraint functions in some sense. Many authors have derived necessary optimality conditions under different constraint qualifications with generalized convexity (see, e.g., [1], [2], [5]-[12], [14], [16], and references therein). Note that if a Lagrange multiplier corresponding to some component of the objective function is equal to zero, then that component has no role in necessary conditions for efficiency. Hence, it is necessary to get Kuhn-Tucker necessary conditions for efficiency with Lagrange multipliers associated with all the components of objective functions to be positive.

Maeda [13] generalizes the Guignard constraints qualification for Fréchet differentiable multiobjective optimization problems consisting of only inequality constraints in finite dimensions, and establishes Kuhn-Tucker necessary

2000 Mathematics Subject Classification: 90C29, 90C46.

Key words and phrases: Theorem of the alternative, Kuhn-Tucker necessary condition, constraint qualification.

Email: dvluu@math.ac.vn (DV Luu), manh-hung.nguyen@malix.univ-paris1.fr (MH Nguyen)

Typeset by $\mathcal{A} \mathcal{M} \mathcal{S}-\mathrm{T}_{\mathrm{E}} \mathrm{X}$ 
conditions for efficiency with positive Lagrange multipliers corresponding to all the components of objective functions. A Maeda type constraint qualification in the semidifferentiable case is considered by Preda-Chitescu [15] for multiobjective mathematical programming involving inequality contraints in finite dimensions. The Kuhn-Tucker necessary conditions for efficiency obtained by Preda-Chitescu [15] are a development further to those due to Maeda [13]. Giorgi-Jiménez-Novo [4] introduce constraint qualifications, which generalize Maeda's constraint qualification [13], and prove an alternative theorem for a system comprising inequalities and equalities together with Kuhn-Tucker necessary conditions for efficiency in finite dimensions in which the positivity of Lagrange multiplies corresponding to all the components of objective functions are ensured.

The purpose of this paper is to develop Kuhn-Tucker necessary conditions for efficiency in multiobjective optimization problems involving inequality, equality and set constraints in normed spaces with positive Lagrange multipliers associated with all the components of objective functions. The remainder of the paper is organized as follows. After some preliminaries, Section 3 presents therems of the alternative for a system involving inequalities, equalities and an inclusion in a normed space, which are generalizations of Tucker's classical theorem of the alternative. In Section 4, some constraint qualifications of Abadie type are proposed and several necessary conditions for efficiency in mathematical programming with inequality, equality and set constraints are established in terms of Dini and Hadamard directional derivatives. Section 5 gives Kuhn-Tucker necessary conditions for efficiency in the problem mentioned above with positive Lagrange multipliers corresponding to all the components of the objective.

\section{Preliminaries}

Let $X$ be a normed space, and let $C$ be a nonempty subset of $X$. Let $f, g$ and $h$ be mappings from $X$ into $\mathbb{R}^{p}, \mathbb{R}^{q}$ and $\mathbb{R}^{r}$, respectively. Note that $f, g, h$ can be naturally expressed as follows: $f=\left(f_{1}, \ldots, f_{p}\right), g=$ $\left(g_{1}, \ldots, g_{q}\right), h=\left(h_{1}, \ldots, h_{r}\right)$, where $f_{k}, g_{j}, h_{\ell}: X \rightarrow \mathbb{R}(k=1, \ldots, p ; j=$ $1, \ldots, q ; \ell=1, \ldots, r)$. In this paper, we shall be concerned with the following 
multiobjective programming problem:

$$
\begin{aligned}
& \min f(x), \\
& \text { subject to } \\
& \qquad \begin{array}{l}
g_{j}(x) \leqslant 0, \quad j=1, \ldots, q ; \\
h_{\ell}(x)=0, \quad \ell=1, \ldots, r ; \\
x \in C .
\end{array}
\end{aligned}
$$

Denote by $M$ the feasible set of (VP)

$$
M=\left\{x \in C: g_{j}(x) \leqslant 0, h_{\ell}(x)=0, j=1, \ldots, q ; \ell=1, \ldots, r\right\} .
$$

Recall that a point $\bar{x} \in M$ is said to be a local efficient solution to Problem $(\mathrm{VP})$ if there exists a number $\delta>0$ such that for all $x \in M \cap B(\bar{x} ; \delta)$,

$$
f(x)-f(\bar{x}) \notin-\mathbb{R}_{+}^{p} \backslash\{0\},
$$

where $R_{+}^{p}$ is the nonnegative orthant of $\mathbb{R}^{p}, B(\bar{x} ; \delta)$ denotes the open ball of radius $\delta$ around $\bar{x}$. This means that $\bar{x} \in M$ is a local efficient solution to (VP) iff there exists $\delta>0$ such that there is no $x \in M \cap B(\bar{x} ; \delta)$ satisfying

$$
\begin{aligned}
f_{k}(x) & \leqslant f_{k}(\bar{x}), \quad k=1, \ldots, p, \\
f_{i}(x) & <f_{i}(\bar{x}) \quad \text { for some } i \in\{1, \ldots, p\} .
\end{aligned}
$$

Recall that the tangent cone (or contingent cone) to $C$ at $\bar{x} \in C$ is the following set:

$$
T(C ; \bar{x})=\left\{v \in X: \exists v_{n} \rightarrow v, \exists t_{n} \downarrow 0 \text { such that } \bar{x}+t_{n} v_{n} \in C, \forall n\right\} .
$$

The cone of sequential linear directions (or sequential radial cone) to $C$ at $\bar{x} \in C$ is the following set:

$$
Z(C ; \bar{x})=\left\{v \in X: \exists t_{n} \downarrow 0 \text { such that } \bar{x}+t_{n} v \in C, \forall n\right\},
$$

where $t_{n} \downarrow 0$ means that $t_{n} \longrightarrow 0^{+}$. Note that both these cones are nonempty. $T(C ; \bar{x})$ is a closed cone, and it may be not convex; $Z(X ; \bar{x}) \subset T(C ; \bar{x})$.

Let $\tilde{f}$ be a real-valued function defined on $X$. The following directional derivatives will be used in the sequel. 
The lower Dini derivative of $\tilde{f}$ at $\bar{x} \in X$ in a direction $v \in X$ is

$$
\underline{D} \widetilde{f}(\bar{x} ; v)=\liminf _{t \downarrow 0} \frac{\widetilde{f}(\bar{x}+t v)-\widetilde{f}(\bar{x})}{t}
$$

The upper Dini derivative of $\tilde{f}$ at $\bar{x}$ in the direction $v$ is

$$
\bar{D} \widetilde{f}(\bar{x} ; v)=\limsup _{t \downarrow 0} \frac{\widetilde{f}(\bar{x}+t v)-\widetilde{f}(\bar{x})}{t} ;
$$

The lower Hadamard derivative of $\widetilde{f}$ at $\bar{x}$ in the direction $v$ is

$$
\underline{d} \tilde{f}(\bar{x} ; v)=\liminf _{\substack{t \downarrow 0 \\ u \rightarrow v}} \frac{\widetilde{f}(\bar{x}+t u)-\widetilde{f}(\bar{x})}{t} ;
$$

The upper Hadamard derivative of $\widetilde{f}$ at $\bar{x}$ in the direction $v$ is

$$
\bar{d} \widetilde{f}(\bar{x} ; v)=\limsup _{\substack{t \downarrow 0 \\ u \rightarrow v}} \frac{\widetilde{f}(\bar{x}+t u)-\widetilde{f}(\bar{x})}{t} .
$$

If $\underline{D} \widetilde{f}(\bar{x} ; v)=\bar{D} \widetilde{f}(\bar{x} ; v)$, we shall denote their common value by $D \widetilde{f}(\bar{x} ; v)$. It is usual directional derivative of $\widetilde{f}$ at $\bar{x}$ in the direction $v$. In case $D \widetilde{f}(\bar{x} ;$. is a continuous linear mapping, $\widetilde{f}$ is said to be Gâteaux differentiable at $\bar{x}$, and

$$
D \widetilde{f}(\bar{x} ; v)=\left\langle\nabla_{G} \widetilde{f}(\bar{x}), v\right\rangle,
$$

where $\nabla_{G} \widetilde{f}(\bar{x})$ denotes the Gâteaux derivative of $\widetilde{f}$ at $\bar{x}$, and $\left\langle\nabla_{G} \widetilde{f}(\bar{x}), v\right\rangle$ is the value of the linear functional $\nabla_{G} \widetilde{f}(\bar{x})$ at the point $v$. Thus if $\widetilde{f}$ is Fréchet differentiable at $\bar{x}$ with Fréchet derivative $\nabla f(\bar{x})$, then

$$
D \widetilde{f}(\bar{x} ; v)=\langle\nabla \widetilde{f}(\bar{x}), v\rangle
$$

Similarly, if $\underline{d} \tilde{f}(\bar{x} ; v)=\bar{d} \widetilde{f}(\bar{x} ; v)$, we also denote their common value by $d \widetilde{f}(\bar{x} ; v)$. This is the Hadamard derivative of $\widetilde{f}$ at $\bar{x}$ in the direction $v$. Note that if $d \widetilde{f}(\bar{x} ; v)$ exists, then also $D \widetilde{f}(\bar{x} ; v)$ exists, and they are equal. 
We set

$$
\begin{aligned}
& I(\bar{x})=\left\{j \in\{1, \ldots, q\}: g_{j}(\bar{x})=0\right\} ; \\
& Q=\left\{x \in C: f_{k}(x) \leqslant f_{k}(\bar{x}), g_{j}(x) \leqslant 0, h_{\ell}(x)=0,\right. \\
& \quad k=1, \ldots, p ; j=1, \ldots, q ; \ell=1, \ldots, r\} ; \\
& Q^{i}=\left\{x \in C: f_{k}(x) \leqslant f_{k}(\bar{x}), g_{j}(x) \leqslant 0, h_{\ell}(x)=0,\right. \\
& \quad k=1, \ldots, p, k \neq i ; j=1, \ldots, q ; \ell=1, \ldots, r\} \quad(i \in\{1, \ldots, p\}) .
\end{aligned}
$$

If for each $v \in Z(C ; \bar{x}), D h_{\ell}(\bar{x} ; v)(\ell=1, \ldots, r)$ exist, we put

$$
\begin{aligned}
C_{D}(Q ; \bar{x})=\{v \in Z(C ; \bar{x}): & \underline{D} f_{k}(\bar{x} ; v) \leqslant 0, k=1, \ldots, p, \\
& \underline{D} g_{j}(\bar{x} ; v) \leqslant 0, j \in I(\bar{x}), \\
D h_{\ell}(\bar{x} ; v) & =0, \ell=1, \ldots, r\} .
\end{aligned}
$$

If for each $v \in T(C ; \bar{x}), d h_{\ell}(\bar{x} ; v)(\ell=1, \ldots, r)$ exists, we put

$$
\begin{aligned}
C_{d}(Q ; \bar{x})=\{v \in T(C ; \bar{x}): & \underline{d} f_{k}(\bar{x} ; v) \leqslant 0, k=1, \ldots, p, \\
& d g_{j}(\bar{x} ; v) \leqslant 0, j \in I(\bar{x}), \\
d h_{\ell}(\bar{x} ; v) & =0, \ell=1, \ldots, r\} .
\end{aligned}
$$

In view of the positive homogeneity of lower Dini and Hadamard directional derivatives, $C_{D}(Q ; \bar{x})$ and $C_{d}(Q ; \bar{x})$ are cones with vertices at the origin.

Let $K$ be a cone in $X$ with vertex at the origin. Denote by $K^{*}$ the dual cone of $K$

$$
K^{*}=\left\{\xi \in X^{*}:\langle\xi, v\rangle \geqslant 0, \forall v \in K\right\},
$$

where $X^{*}$ is the topological dual of $X$. Note that $K^{*}$ is a weakly* closed convex cone.

In what follows we recall three results in [3] (Lemma 5.8, Lemma 5.11 and Theorem 10.4), which will be used in the proofs of Theorems 3.1 and 3.2 below.

Proposition 2.1 [3]. Let $K_{\alpha}(\alpha \in I)$ be weakly closed convex cones in $X$. Assume that $\sum_{\alpha \in I} K_{\alpha}^{*}$ is weakly closed. Then,

$$
\left(\bigcap_{\alpha \in I} K_{\alpha}\right)^{*}=\sum_{\alpha \in I} K_{\alpha}^{*}
$$


Proposition 2.2 [3] (Dubovitskii-Milyutin Theorem). Assume that $K_{1}, \ldots$, $K_{n}, K_{n+1}$ are convex cones with vertices at the origin in $X$, and $K_{1}, \ldots, K_{n}$ are open. Then, $\bigcap_{i=1}^{n+1} K_{i}=\emptyset$ if and only if there exist $\xi_{i} \in K_{i}^{*}(i=1, \ldots, n+1)$, not all zero, such that

$$
\xi_{1}+\cdots+\xi_{n}+\xi_{n+1}=0
$$

Proposition 2.3 [3] (Farkas-Minkowski Theorem). Let

$$
K=\left\{x \in \mathbb{R}^{m}:\left\langle a_{i}, x\right\rangle \geqslant 0, a_{i} \in \mathbb{R}^{m}, i=1, \ldots, n\right\} .
$$

Then,

$$
K^{*}=\left\{\sum_{i=1}^{n} a_{i} y_{i}: y_{i} \geqslant 0, i=1, \ldots, n\right\}
$$

\section{Theorems of the Alternative}

In order to derive Kuhn-Tucker necessary conditions for efficiency, in this section we investigate theorems of the alternative for a system consisting of inequalities, equalities and an inclusion.

Let $X$ be a normed space with the topological dual $X^{*}$. Let $a_{k}, b_{j}, c_{\ell}$ be vectors in $X^{*}(k=1, \ldots, p ; j=1, \ldots, q ; \ell=1, \ldots, r)$, and let $C$ be a nonempty subset of $X$. For $i \in\{1, \ldots, p\}$, we set

$$
\begin{aligned}
& A_{k}=\left\{v \in X:\left\langle a_{k}, v\right\rangle \leqslant 0\right\} \quad(k=1, \ldots, p ; k \neq i), \\
& \widetilde{A}_{i}=\left\{v \in X:\left\langle a_{i}, v\right\rangle<0\right\}, \\
& B_{j}=\left\{v \in X:\left\langle b_{j}, v\right\rangle \leqslant 0\right\} \quad(j=1, \ldots, s), \\
& C_{\ell}=\left\{v \in X:\left\langle c_{\ell}, v\right\rangle=0\right\} \quad(\ell=1, \ldots, r) .
\end{aligned}
$$

Note that $A_{k}$ and $B_{j}(k=1, \ldots, p, k \neq i ; j=1, \ldots, s)$ are closed convex cones with vertices at the origin, $\widetilde{A}_{i}$ is an open convex cone with vertex at the origin, and $C_{\ell}(\ell=1, \ldots, r)$ are closed linear subspaces of $X$.

Theorem 3.1. Assume that

(a) $K$ is an arbitrary nonempty convex subcone of $T(C ; \bar{x})$ with vertex at the origin, and $K$ is closed; 
(b) For each $i \in\{1, \ldots, p\}$, the set

$$
\sum_{\substack{k=1 \\ k \neq i}}^{p} A_{k}^{*}+\sum_{j=1}^{s} B_{j}^{*}+\sum_{\ell=1}^{r} C_{\ell}^{*}+K^{*}
$$

is weakly* closed in $X^{*}$.

Then exactly one of the following two conclusions holds:

(i) For each $i \in\{1, \ldots, p\}$, the system

$$
\begin{aligned}
& \left\langle a_{k}, v\right\rangle \leqslant 0, \quad k=1, \ldots, p ; k \neq i \\
& \left\langle a_{i}, v\right\rangle<0, \\
& \left\langle b_{j}, v\right\rangle \leqslant 0, \quad j=1, \ldots, s, \\
& \left\langle c_{\ell}, v\right\rangle=0, \quad \ell=1, \ldots, r, \\
& v \in K,
\end{aligned}
$$

has no solution $v \in X$.

(ii) There exist $\bar{\lambda}_{k}>0(k=1, \ldots, p), \bar{\mu}_{j} \geqslant 0(j=1, \ldots, s)$ and $\bar{\nu}_{\ell} \in \mathbb{R}$ $(\ell=1, \ldots, r)$ such that

$$
\sum_{k=1}^{p} \bar{\lambda}_{k}\left\langle a_{k}, v\right\rangle+\sum_{j=1}^{s} \bar{\mu}_{j}\left\langle b_{j}, v\right\rangle+\sum_{\ell=1}^{r} \bar{\nu}_{\ell}\left\langle c_{\ell}, v\right\rangle \geqslant 0 \quad(\forall v \in K) .
$$

Remark 3.1. If assumption (a) is replaced by that $K$ is a convex nonempty subcone of $Z(C ; \bar{x})$ and $K$ is closed, then Theorem 3.1 is still valid, since $Z(C ; \bar{x}) \subset T(C ; \bar{x})$.

\section{Proof of Theorem 3.1.}

(i) $\Rightarrow$ (ii): We need only consider the case of all $a_{k} \neq 0(k=1, \ldots, p)$, since in case there exists $a_{k_{0}}=0$, we shall take $\bar{\lambda}_{k_{0}}=1$. For each $i \in\{1, \ldots, p\}$, suppose that the system (1)-(5) has no solution $v \in X$. Putting

$$
D_{i}=\left(\bigcap_{\substack{k=1 \\ k \neq i}}^{p} A_{k}\right) \cap\left(\bigcap_{j=1}^{s} B_{j}\right) \cap\left(\bigcap_{\ell=1}^{r} C_{\ell}\right) \cap K,
$$

we can see that $D_{i}$ is a nonempty closed convex cone in $X$ with vertex at the origin, and

$$
\widetilde{A}_{i} \cap D_{i}=\emptyset .
$$


Note that $\widetilde{A}_{i}$ is a nonempty convex cone with vertex at the origin, as $a_{i} \neq 0$.

We invoke Proposition 2.2 to deduce that there exist $\xi_{i} \in \widetilde{A}_{i}^{*}$ and $\eta_{i} \in D_{i}^{*}$, not all zero, such that

$$
\xi_{i}+\eta_{i}=0
$$

If follows readily from $(7)$ that $\xi_{i} \neq 0$ (also $\eta_{i} \neq 0$ ). Since the convex cones $A_{k}$, $B_{j}, C_{\ell}(k=1, \ldots, p, k \neq i ; j=1, \ldots, s ; \ell=1, \ldots, r)$ and $K$ are closed, they are weakly closed. Thus all the hypotheses of Proposition 2.1 are fulfilled. Taking account of Proposition 2.1, we get

$$
D_{i}^{*}=\sum_{\substack{k=1 \\ k \neq i}}^{p} A_{k}^{*}+\sum_{j=1}^{s} B_{j}^{*}+\sum_{\ell=1}^{r} C_{\ell}^{*}+K^{*}
$$

On the other hand, in view of Theorem 10.2 in [3] on dual cones, we have

$$
\begin{aligned}
A_{k}^{*} & =\left\{\lambda a_{k}: \lambda \leqslant 0\right\}, \quad k=1, \ldots, p ; k \neq i ; \\
\widetilde{A}_{i}^{*} & =\left\{\lambda a_{i}: \lambda \leqslant 0\right\} \quad\left(\text { as } a_{i} \neq 0\right) ; \\
B_{j}^{*} & =\left\{\mu b_{j}: \mu \leqslant 0\right\}, \quad j=1, \ldots, s ; \\
C_{\ell}^{*} & =\left\{\nu c_{\ell}: \nu \in \mathbb{R}\right\}, \quad \ell=1, \ldots, r .
\end{aligned}
$$

Since $\xi_{i} \in \widetilde{A}_{i}^{*}, \xi_{i} \neq 0$, it follows that $\xi_{i}=\lambda_{i} a_{i}$ with $\lambda_{i}<0$. By virtue of (8), there exists $\lambda_{i k} \leqslant 0(k=1, \ldots, p ; k \neq i), \mu_{i j} \leq 0(j=1, \ldots, s), \nu_{i \ell} \in \mathbb{R}$ $(\ell=1, \ldots, r)$ and $\zeta_{i} \in K^{*}$ such that

$$
\eta_{i}=\sum_{\substack{k=1 \\ k \neq i}}^{p} \lambda_{i k} a_{k}+\sum_{j=1}^{s} \mu_{i j} b_{j}+\sum_{\ell=1}^{r} \nu_{i \ell} c_{\ell}+\zeta_{i}
$$

Setting $\bar{\lambda}_{i k}=-\lambda_{i k}(k=1, \ldots, p ; k \neq i), \bar{\lambda}_{i i}=-\lambda_{i}, \bar{\mu}_{i j}=-\mu_{i j}(j=$ $1, \ldots, s), \bar{\nu}_{i \ell}=-\nu_{i \ell}(\ell=1, \ldots, r)$, one gets that $\bar{\lambda}_{i k} \geqslant 0(k=1, \ldots p ; k \neq i)$, $\bar{\lambda}_{i, i}>0, \bar{\mu}_{i j} \geqslant 0(j=1, \ldots, s)$, and $\bar{\nu}_{i \ell} \in \mathbb{R}(\ell=1, \ldots, r)$. It follows from $(7)$ that

$$
\sum_{k=1}^{p} \bar{\lambda}_{i k} a_{k}+\sum_{j=1}^{s} \bar{\mu}_{i j} b_{j}+\sum_{\ell=1}^{r} \bar{\nu}_{i \ell} c_{j}=\zeta_{i} \in K^{*}
$$

which implies that

$$
\sum_{k=1}^{p} \bar{\lambda}_{i k}\left\langle a_{k}, v\right\rangle+\sum_{j=1}^{s} \bar{\mu}_{i j}\left\langle b_{j}, v\right\rangle+\sum_{\ell=1}^{r} \bar{\nu}_{i \ell}\left\langle c_{j}, v\right\rangle \geqslant 0 \quad(\forall v \in K) .
$$


Note that for each $i \in\{1, \ldots, p\}$, we obtain the inequality (9i). Adding up both sides of $(9 \mathrm{i}), i=1, \ldots, p$, and putting $\bar{\lambda}_{k}=\sum_{i=1}^{p} \bar{\lambda}_{i k}, \bar{\mu}_{j}=\sum_{i=1}^{p} \bar{\mu}_{i j}$ and $\bar{\nu}_{\ell}=\sum_{i=1}^{p} \nu_{i \ell}$, we obtain that $\bar{\lambda}_{k}>0, \bar{\mu}_{j} \geqslant 0, \bar{\nu}_{\ell} \in \mathbb{R}(k=1, \ldots, p ; j=$ $1, \ldots, s ; \ell=1, \ldots, r)$, and

$$
\sum_{k=1}^{p} \bar{\lambda}_{k}\left\langle a_{k}, v\right\rangle+\sum_{j=1}^{s} \bar{\mu}_{j}\left\langle b_{j}, v\right\rangle+\sum_{\ell=1}^{r} \bar{\nu}_{\ell}\left\langle c_{\ell}, v\right\rangle \geqslant 0 \quad(\forall v \in K) .
$$

(ii) $\Rightarrow$ (i): Suppose that there exist $\bar{\lambda}_{k}>0, \bar{\mu}_{j} \geqslant 0$ and $\bar{\nu}_{\ell} \in \mathbb{R}(k=$ $1, \ldots, p ; j=1, \ldots, s ; \ell=1, \ldots, r)$ satisfying (6). If (i) were false, there would exist $i \in\{1, \ldots, p\}$ such that the system (1)-(5) has a solution $v_{0} \in X$. It follows from this that

$$
\sum_{k=1}^{p} \bar{\lambda}_{k}\left\langle a_{k}, v_{0}\right\rangle+\sum_{j=1}^{s} \bar{\mu}_{j}\left\langle b_{j}, v_{0}\right\rangle+\sum_{\ell=1}^{r} \bar{\nu}_{\ell}\left\langle c_{\ell}, v_{0}\right\rangle<0,
$$

which contradicts (6). The proof is complete.

Corollary 3.1. Suppose that $C$ is convex, and for each $i \in\{1, \ldots, p\}$ the set

$$
\sum_{\substack{k=1 \\ k \neq i}}^{p} A_{k}^{*}+\sum_{j=1}^{s} B_{j}^{*}+\sum_{\ell=1}^{r} C_{\ell}^{*}+T(X ; \bar{x})^{*}
$$

is weakly* closed in $X^{*}$. Then exactly one of the following two conclusions holds:

(i') For each $i \in\{1, \ldots, p\}$, the system (1)-(5), in which $K$ is replaced by $T(C ; \bar{x})$, has no solution $v \in X$.

(ii') There exist $\bar{\lambda}_{k}>0, \bar{\mu}_{j} \geqslant 0, \bar{\nu}_{\ell} \in \mathbb{R}(k=1, \ldots, p ; j=1, \ldots, s ; \ell=$ $1, \ldots, r)$ such that

$$
\sum_{k=1}^{p} \bar{\lambda}_{k}\left\langle a_{k}, v\right\rangle+\sum_{j=1}^{s} \bar{\mu}_{j}\left\langle b_{j}, v\right\rangle+\sum_{\ell=1}^{r} \bar{\nu}_{\ell}\left\langle c_{\ell}, v\right\rangle \geqslant 0 \quad(\forall v \in T(C ; \bar{x})) .
$$

Proof. Since $C$ is nonempty convex, $T(C ; \bar{x})$ is a nonempty closed convex cone. Applying Theorem 3.1 to $K=T(C ; \bar{x})$, we obtain the desired assertion of Corrollary 3.1. 
For each $i \in\{1, \ldots, p\}$, we set

$$
E_{i}=\left(\bigcap_{\substack{k=1 \\ k \neq i}}^{p} A_{k}\right) \cap\left(\bigcap_{j=1}^{s} B_{j}\right) \cap\left(\bigcap_{\ell=1}^{r} C_{\ell}\right) .
$$

It is obvious that $E_{i}$ is a nonempty closed cone with vertex at the origin.

In case $\operatorname{dim} X<+\infty$, with the help of the Farkas-Minkowski theorem, condition (b) in Theorem 3.1 will be replaced by a weakened condition as in the following theorem.

Theorem 3.2. Let $\operatorname{dim} X<+\infty$, and let $K$ be a nonempty convex subcone of $T(C ; \bar{x})$ with vertex at the origin, and $K$ closed. Assume that for each $i \in\{1, \ldots, p\}$, the set $E_{i}^{*}+K^{*}$ is closed. Then exactly one of the following two conclusions holds:

(!) For each $i \in\{1, \ldots, p\}$, the system (1)-(5) has no solution $v \in X$.

(!!) There exists $\bar{\lambda}_{k}>0, \bar{\mu}_{j} \geqslant 0$ and $\bar{\nu}_{\ell} \in \mathbb{R}(k=1, \ldots, p ; j=1, \ldots, s ; \ell=$ $1, \ldots, r)$ such that (6) holds.

Proof. Since $\operatorname{dim} X<+\infty$, it holds that $\operatorname{dim} X^{*}=\operatorname{dim} X$, and so all the topologies on $X^{*}$ conside. Making use of Proposition 2.3, we deduce that for each $i \in\{1, \ldots, p\}$,

$$
\begin{array}{r}
E_{i}^{*}=\left\{\sum_{\substack{k=1 \\
k \neq i}}^{p} \lambda_{i k} a_{k}+\sum_{j=1}^{s} \mu_{i j} b_{j}+\sum_{\ell=1}^{r} \nu_{i \ell} c_{\ell}: \lambda_{i k} \leqslant 0, \mu_{i j} \leqslant 0, \nu_{i \ell} \in \mathbb{R},\right. \\
k=1, \ldots, p, k \neq i ; j=1, \ldots, s ; \ell=1, \ldots, r\},
\end{array}
$$

which leads to the following

$$
E_{i}^{*}=\sum_{\substack{k=1 \\ k \neq i}}^{p} A_{k}^{*}+\sum_{j=1}^{s} B_{j}^{*}+\sum_{\ell=1}^{r} C_{\ell}^{*} \quad(i=1, \ldots, p) .
$$

So, by assumption, the set

$$
\sum_{\substack{k=1 \\ k \neq i}}^{p} A_{k}^{*}+\sum_{j=1}^{s} B_{j}^{*}+\sum_{\ell=1}^{r} C_{\ell}^{*}+K^{*}
$$

is closed. Thus all the hypotheses of Theorem 3.1 are fulfilled, and hence from Theorem 3.1 the conclusion follows.

In case $\operatorname{dim} X<+\infty$ and $C=X$, from Theorem 3.2 we can obtain Tucker's classical theorem of the alternative (see, e.g., [14]) as a special case. 
Corollary 3.2. Let $\operatorname{dim} X<+\infty$. Then exactly one of the following two assertions holds:

(!') For each $i \in\{1, \ldots, p\}$, the system (1)-(4) has no solution $v \in X$.

(!!') There exists $\bar{\lambda}_{k}>0, \bar{\mu}_{j} \geqslant 0, \bar{\nu}_{\ell} \in \mathbb{R}(k=1, \ldots, p ; j=1, \ldots, s ; \ell=$ $1, \ldots, r)$ such that

$$
\sum_{k=1}^{p} \bar{\lambda}_{k} a_{k}+\sum_{j=1}^{s} \bar{\mu}_{j} b_{j}+\sum_{\ell=1}^{r} \bar{\nu}_{\ell} c_{\ell}=0
$$

Proof. For $C=X$, it results that $T(C ; \bar{x})=X$, and hence $T(C ; \bar{x})^{*}=\{0\}$. Moreover, since $\operatorname{dim} X<+\infty$, for each $i \in\{1, \ldots, p\}, E_{i}^{*}$ is a nonempty closed convex cone in $X^{*}$, and $0 \in E_{i}^{*}$. Hence, $E_{i}^{*}+T(C ; \bar{x})^{*}=E_{i}^{*}$, and so $E_{i}^{*}+T(C ; \bar{x})^{*}$ is closed in $X^{*}$. We now apply Theorem 3.2 to $C=X$ and deduce that (!') is equivalent to that there exists $\bar{\lambda}_{k}>0, \bar{\mu}_{j} \geqslant 0, \bar{\nu}_{\ell} \in \mathbb{R}$ $(k=1, \ldots, p ; j=1, \ldots, s ; \ell=1, \ldots, r)$ such that

$$
\sum_{k=1}^{p} \bar{\lambda}_{k}\left\langle a_{k}, v\right\rangle+\sum_{j=1}^{s} \bar{\mu}_{j}\left\langle b_{j}, v\right\rangle+\sum_{\ell=1}^{r} \bar{\nu}_{\ell}\left\langle c_{\ell}, v\right) \geqslant 0 \quad(\forall v \in T(X ; \bar{x})=X),
$$

which is equivalent to (10).

\section{Constraint qualifications And neCessary CONDitions FOR EFFICIENCY}

We now turn to Problem $(\mathrm{P})$. Hereafter we shall introduce two constraint qualifications of Abadie type in terms of Dini and Hadamard directional derivatives and derive necessary conditions for efficiency.

Proposition 4.1. Let $\bar{x} \in M$.

a) If for each $v \in T(C ; \bar{x})$, the Hadamard directional derivatives $d h_{1}(\bar{x} ; v)$, $\ldots, d h_{r}(\bar{x} ; v)$ exist, then

$$
\bigcap_{i=1}^{p} T\left(Q^{i} ; \bar{x}\right) \subset C_{d}(Q ; \bar{x}) .
$$

b) If for each $v \in Z(C ; \bar{x})$, the Dini directional derivatives $D h_{1}(\bar{x} ; v), \ldots$, $D h_{r}(\bar{x} ; v)$ exists, then

$$
\bigcap_{i=1}^{p} Z\left(Q^{i} ; \bar{x}\right) \subset C_{D}(Q ; \bar{x})
$$


Proof. We shall only prove (11), while (12) is analogously treated. We begin with showing that for $i=1, \ldots, p$,

$$
T\left(Q^{i} ; \bar{x}\right) \subset C_{d}\left(Q^{i} ; \bar{x}\right),
$$

where

$$
\begin{aligned}
& C_{d}\left(Q^{i}, \bar{x}\right)=\{v \in T(C ; \bar{x}): \underline{d} f_{k}(\bar{x} ; v) \leqslant 0, k=1, \ldots, p ; k \neq i, \\
& \underline{d} g_{j}(\bar{x} ; v) \leqslant 0, \quad j \in I(\bar{x}), \\
&\left.d h_{\ell}(\bar{x} ; v)=0, \ell=1, \ldots, r\right\} .
\end{aligned}
$$

For $i \in\{1, \ldots, p\}$, taking $v \in T\left(Q^{i} ; \bar{x}\right)$, there exist $t_{n} \downarrow 0$ and $v_{n} \rightarrow v$ such that $\bar{x}+t_{n} v_{n} \in Q^{i}(\forall n)$. Then $\bar{x}+t_{n} v_{n} \in C(\forall n)$, and so $v \in T(C ; \bar{x})$.

Moreover, for $i \in\{1, \ldots, p\}$, since $\bar{x}+t_{n} v_{n} \in Q^{i}$, it holds that

$$
\begin{array}{ll}
f_{k}\left(\bar{x}+t_{n} v_{n}\right) \leqslant f_{k}(\bar{x}), \quad k=1, \ldots, p ; k \neq i ; \\
g_{j}\left(\bar{x}+t_{n} v_{n}\right) \leqslant 0=g_{j}(\bar{x}), \quad j \in I(\bar{x}) ; \\
h_{\ell}\left(\bar{x}+t_{n} v_{n}\right)=0=h_{\ell}(\bar{x}), \quad \ell=1, \ldots, r .
\end{array}
$$

Consequently,

$$
\begin{aligned}
& \underline{d} f_{k}(\bar{x} ; v) \leqslant \liminf _{n \rightarrow \infty} \frac{f_{k}\left(\bar{x}+t_{n} v_{n}\right)-f_{k}(\bar{x})}{t_{n}} \leqslant 0, \quad k=1, \ldots, p ; k \neq i ; \\
& \underline{d} g_{j}(\bar{x} ; v) \leqslant \liminf _{n \rightarrow \infty} \frac{g_{j}\left(\bar{x}+t_{n} v_{n}\right)-g_{j}(\bar{x})}{t_{n}} \leqslant 0, \quad j \in I(\bar{x}) ; \\
& d h_{\ell}(\bar{x} ; v)=\lim _{n \rightarrow \infty} \frac{h_{\ell}\left(\bar{x}+t_{n} v_{n}\right)-h_{\ell}(\bar{x})}{t_{n}}=0, \quad \ell=1, \ldots, r .
\end{aligned}
$$

Hence, $v \in C_{d}\left(Q^{i} ; \bar{x}\right)$. Thus we already arrive at (13). It follows from (13) that

$$
\bigcap_{i=1}^{p} T\left(Q^{i} ; \bar{x}\right) \subset \bigcap_{i=1}^{p} C_{d}\left(Q^{i} ; \bar{x}\right)=C_{d}(Q ; \bar{x}),
$$

as was to be shown.

Note that the converse inclusions of (11) and (12) do not in general hold. Hence, in order to derive necessary conditions for efficiency in Problem (VP), 
it is reasonable to introduce the following constraint qualifications of Abadie type at $\bar{x}$ :

$$
\begin{aligned}
C_{d}(Q ; \bar{x}) & \subset \bigcap_{i=1}^{p} T\left(Q^{i} ; \bar{x}\right), \\
C_{D}(Q ; \bar{x}) & \subset \bigcap_{i=1}^{p} Z\left(Q^{i} ; \bar{x}\right) .
\end{aligned}
$$

They are generalizations of the generalized Abadie constraint qualifications in [4], [13], [15].

If for each $v \in T(C ; \bar{x})$, the Hadamard directional derivatives $d f_{k}(\bar{x} ; v)$ and $d h_{\ell}(\bar{x} ; v)(k=1, \ldots, p ; \ell=1, \ldots, r)$ exist, for $i \in\{1, \ldots, p\}$, we set

$$
\begin{aligned}
& L_{d}^{i}(f ; \bar{x})=\left\{v \in T(C ; \bar{x}): d f_{i}(\bar{x} ; v)<0, d f_{k}(\bar{x} ; v) \leqslant 0, k=1, \ldots, p ; k \neq i\right\}, \\
& L_{d}(M ; \bar{x})=\left\{v \in T(C ; \bar{x}): \underline{d} g_{j}(\bar{x} ; v) \leqslant 0, j \in I(\bar{x})\right. \text {, } \\
& \left.d h_{\ell}(\bar{x} ; v)=0, \ell=1, \ldots, r\right\},
\end{aligned}
$$

where $M$ denotes the feasible set of Problem (VP).

A necessary condition for efficiency can be stated as follows.

Theorem 4.1. Let $\bar{x}$ be a local efficient solution to Problem (VP). Assume that the function $g_{j}(j \notin I(\bar{x}))$ are continuous at $\bar{x}$, and for each $v \in T(C ; \bar{x})$, the Hadamard directional derivatives $d f_{k}(\bar{x} ; v)$ and $d h_{\ell}(\bar{x}, v)(k=1, \ldots, p ; \ell=$ $1, \ldots, r)$ exist. Suppose, in addition, that the constraint qualification (14) holds at $\bar{x}$. Then, for each $i \in\{1, \ldots, p\}$,

$$
L_{d}^{i}(f ; \bar{x}) \cap L_{d}(M ; \bar{x})=\emptyset .
$$

Proof. Assume the contrary, that there exists $i_{0} \in\{1, \ldots, p\}$ such that

$$
L_{d}^{i_{0}}(f ; \bar{x}) \cap L_{d}(M ; \bar{x}) \neq \emptyset
$$

which implies that there exists $v_{0} \in L_{d}^{i_{0}}(f ; \bar{x}) \cap L_{d}(M ; \bar{x})$. Since $v_{0} \in L_{d}^{i_{0}}(f ; \bar{x})$, it holds that

$$
\begin{aligned}
& d f_{i_{0}}\left(\bar{x} ; v_{0}\right)<0, \\
& d f_{k}\left(\bar{x} ; v_{0}\right) \leqslant 0, \quad k=1, \ldots, p ; k \neq i_{0} .
\end{aligned}
$$


It is obvious that $v_{0} \in C_{d}(Q ; \bar{x})$. Making use of the constraint qualification (14), we get that $v_{0} \in \bigcap_{i=1}^{p} T\left(Q^{i} ; \bar{x}\right)$, and so $v_{0} \in T\left(Q^{i_{0}} ; \bar{x}\right)$. Therefore, there exist sequence $t_{n} \downarrow 0$ and $v_{n} \rightarrow v_{0}$ such that $\bar{x}+t_{n} v_{n} \in Q^{i_{0}}(\forall n)$. Hence, $\bar{x}+t_{n} v_{n} \in C$, and

$$
\begin{aligned}
& f_{k}\left(\bar{x}+t_{n} v_{n}\right) \leqslant f_{k}(\bar{x}), \quad k=1, \ldots, p ; k \neq i_{0} \\
& g_{j}\left(\bar{x}+t_{n} v_{n}\right) \leqslant 0, \quad j \in I(\bar{x}) \\
& h_{\ell}\left(\bar{x}+t_{n} v_{n}\right)=0, \quad \ell=1, \ldots, r .
\end{aligned}
$$

Moreover, for $j \notin I(\bar{x})$, one has $g_{j}(\bar{x})<0$. In view of the continuity of $g_{j}(j \notin I(\bar{x}))$, there exists a natural number $N_{1}$ such that for all $n \geqslant N_{1}$, $g_{j}\left(\bar{x}+t_{n} v_{n}\right) \leqslant 0 \quad(j \notin I(\bar{x}))$.

On the other hand, since $\bar{x}$ is a local efficiency solution of Problem (VP), there exists a number $\delta>0$ such that there is no $x \in M \cap B(\bar{x} ; \delta)$ satisfying

$$
\begin{aligned}
f_{k}(x) \leq f_{k}(\bar{x}), & k=1, \ldots, p, \\
f_{i}(x)<f_{i}(\bar{x}) & \text { for some } i \in\{1, \ldots, p\} .
\end{aligned}
$$

From the proofs above, it follows that there is a natural number $N\left(\geqslant N_{1}\right)$ such that for all $n \geqslant N, \bar{x}+t_{n} v_{n} \in M \cap B(\bar{x} ; \delta)$. Consequently, for all $n \geqslant N$,

$$
f_{i_{0}}\left(\bar{x}+t_{n} v_{n}\right)>f_{i_{0}}(\bar{x}),
$$

which leads to the following

$$
d f_{i_{0}}\left(\bar{x} ; v_{0}\right) \geqslant 0 \text {. }
$$

This conflicts with (17). Hence, for each $i \in\{1, \ldots, p\},(16)$ holds.

Remark 4.1. Theorem 4.1 is a generalization of Theorem 3.1 in [13].

If for each $v \in Z(C ; \bar{x})$, the Dini directional derivatives $D f_{k}(\bar{x} ; v)$ and $D h_{\ell}(\bar{x} ; v)(k=1, \ldots, p ; \ell=1, \ldots, r)$ exist, for $i \in\{1, \ldots, p\}$, we set

$$
\begin{aligned}
& L_{D}^{i}(f ; \bar{x})=\left\{v \in Z(C ; \bar{x}): D f_{i}(\bar{x} ; v)<0, D f_{k}(\bar{x} ; v) \leqslant 0, k=1, \ldots, p ; k \neq i\right\}, \\
& L_{D}(M ; \bar{x})=\left\{v \in Z(C ; \bar{x}): \underline{D g_{j}(\bar{x} ; v)} \leqslant 0, j \in I(\bar{x}),\right. \\
&\left.D h_{\ell}(\bar{x} ; v)=0, \ell=1, \ldots, r\right\} .
\end{aligned}
$$

By an argument analogous to that used for the proof of Theorem 4.1, we obtain the following 
Theorem 4.2. Let $\bar{x}$ be a local efficient solution of Problem (VP). Assume that the functions $g_{j}(j \notin I(\bar{x}))$ are continuous at $\bar{x}$, and for each $v \in Z(C ; \bar{x})$, the Dini directional derivatives $D f_{k}(\bar{x} ; v)$ and $D h_{\ell}(\bar{x} ; v)(k=1, \ldots, p ; \ell=$ $1, \ldots, r)$ exist. Suppose. furthermore, that the constraint qualification (15) holds at $\bar{x}$. Then, for each $i \in\{1, \ldots, p\}$,

$$
L_{D}^{i}(f ; \bar{x}) \cap L_{D}(M ; \bar{x})=\emptyset .
$$

\section{Kuhn-TuCKeR NECESSARY CONDITIONS FOR EFFICIENCY}

In this section, turning back Problem (VP), we suppose that the functions $f_{k}, g_{j}$ and $h_{\ell}$ are Gâteaux differentiable at $\bar{x}$ with Gâteaux derivatives $\nabla_{G} f_{k}(\bar{x}), \nabla_{G} g_{j}(\bar{x})$ and $\nabla_{G} h_{\ell}(\bar{x})(k=1, \ldots, p ; j=1, \ldots, q ; \ell=1, \ldots, r)$, and the functions $g_{j}(j \notin I(\bar{x}))$ are continuous. Then, for each $v \in X$

$$
\begin{aligned}
& d f_{k}(\bar{x} ; v)=D f_{k}(\bar{x} ; v)=\left\langle\nabla_{G} f_{k}(\bar{x}), v\right\rangle \quad(k=1, \ldots, p), \\
& d g_{j}(\bar{x} ; v)=D g_{j}(\bar{x} ; v)=\left\langle\nabla_{G} g_{j}(\bar{x}), v\right\rangle \quad(j=1, \ldots, q), \\
& d h_{\ell}(\bar{x} ; v)=D h_{\ell}(\bar{x} ; v)=\left\langle\nabla_{G} h_{\ell}(\bar{x}), v\right\rangle \quad(\ell=1, \ldots, r), \\
& \text { and } \quad C_{d}(Q ; \bar{x})=\left\{v \in T(C ; \bar{x}):\left\langle\nabla_{G} f_{k}(\bar{x}), v\right\rangle \leqslant 0, k=1, \ldots, p,\right. \\
&\left\langle\nabla_{G} g_{j}(\bar{x}), v\right\rangle \leqslant 0, j \in I(\bar{x}), \\
&\left.\left\langle\nabla_{G} h_{\ell}(\bar{x}), v\right\rangle=0, \ell=1, \ldots, r\right\} .
\end{aligned}
$$

Note that if the functions $g_{j}(j \notin I(\bar{x}))$ only are Gâteaux differentiable at $\bar{x}$, then they are not necessarily continuous at $\bar{x}$. Using the notations $A_{k}, \widetilde{A}_{i}, B_{j}$, $C_{\ell}$ as in Section 3, and taking $a_{k}=\nabla_{G} f_{k}(\bar{x}), b_{j}=\nabla_{G} g_{j}(\bar{x}), c_{\ell}=\nabla_{G} h_{\ell}(\bar{x})$ $(k=1, \ldots, p ; j \in I(\bar{x}) ; \ell=1, \ldots, r)$, we obtain the following Kuhn-Tucker necessary conditions for efficiency to (VP) in which Lagrange multipliers associated with all the components of the objective are positive.

Theorem 5.1. Let $\bar{x}$ be a local efficient slution to Problem (VP), and let $K$ be an arbitrary nonempty convex subcone of $T(C ; \bar{x})$ with vertex at the origin and $K$ closed. Assume that for each $i \in\{1, \ldots, p\}$, the set

$$
\sum_{\substack{k=1 \\ k \neq i}}^{p} A_{k}^{*}+\sum_{j \in I(\bar{x})} B_{j}^{*}+\sum_{\ell=1}^{r} C_{\ell}^{*}+K^{*}
$$


is weakly* closed in $X^{*}$. Suppose also that the constraint qualification (14) holds at $\bar{x}$. Then there exist $\bar{\lambda}_{k}>0, \bar{\mu}_{j} \geqslant 0$ and $\bar{\nu}_{\ell} \in \mathbb{R}(k=1, \ldots, p ; j=$ $1, \ldots, q ; \ell=1, \ldots, r)$ such that

$$
\begin{gathered}
\sum_{k=1}^{p} \bar{\lambda}_{k}\left\langle\nabla_{G} f_{k}(\bar{x}), v\right\rangle+\sum_{j=1}^{q} \bar{\mu}_{j}\left\langle\nabla_{G} g_{j}(\bar{x}), v\right\rangle+\sum_{\ell=1}^{r} \bar{\nu}_{\ell}\left\langle\nabla_{G} h_{\ell}(\bar{x}), v\right\rangle \geqslant 0 \quad(\forall v \in K), \\
\bar{\mu}_{j} g_{j}(\bar{x})=0 \quad(j=1, \ldots, q) .
\end{gathered}
$$

Proof. We invoke Theorem 4.1 to deduce that for each $i \in\{1, \ldots, p\}$, the system

$$
\begin{aligned}
& \left\langle\nabla_{G} f_{k}(\bar{x}), v\right\rangle \leqslant 0, \quad k=1, \ldots, p ; k \neq i \\
& \left\langle\nabla_{G} f_{i}(\bar{x}), v\right\rangle<0, \\
& \left\langle\nabla_{G} g_{j}(\bar{x}), v\right\rangle \leqslant 0, \quad j \in I(\bar{x}) \\
& \left\langle\nabla_{G} h_{\ell}(\bar{x}), v\right\rangle=0, \quad \ell=1, \ldots, r \\
& v \in K
\end{aligned}
$$

has no solution $v \in X$.

Applying Theorem 3.1 to $a_{k}=\nabla_{G} f_{k}(\bar{x}), b_{j}=\nabla_{G} g_{j}(\bar{x}), c_{\ell}=\nabla_{G} h_{\ell}(\bar{x})$ $(k=1, \ldots, p ; j \in I(\bar{x}) ; \ell=1, \ldots, r)$ yields the existence of $\bar{\lambda}_{k}>0, \bar{\mu}_{j} \geqslant 0$ and $\bar{\nu}_{\ell} \in \mathbb{R}(k=1, \ldots, p ; j \in I(\bar{x}) ; \ell=1, \ldots, r)$ satisfying

$\sum_{k=1}^{p} \bar{\lambda}_{k}\left\langle\nabla_{G} f_{k}(\bar{x}), v\right\rangle+\sum_{j \in I(\bar{x})} \bar{\mu}_{j}\left\langle\nabla_{G} g_{j}(\bar{x}), v\right\rangle+\sum_{\ell=1}^{r} \bar{\nu}_{\ell}\left\langle\nabla_{G} h_{\ell}(\bar{x}), v\right\rangle \geqslant 0(\forall v \in K)$.

For $j \notin I(\bar{x})$, we take $\bar{\mu}_{j}=0$ and obtain (18). Moreover, we also get (19), because for $j \in I(\bar{x}), g_{j}(\bar{x})=0$ and for $j \notin I(\bar{x}), \mu_{j}=0$.

Corollary 5.1. Let $\bar{x}$ be a local efficient solution of Problem (VP), and $C$ convex. Assume that for each $i \in\{1, \ldots, p\}$, the set

$$
\sum_{\substack{k=1 \\ k \neq i}}^{p} A_{k}^{*}+\sum_{j \in I(\bar{x})} B_{j}^{*}+\sum_{\ell=1}^{r} C_{\ell}^{*}+T(C ; \bar{x})^{*}
$$

is weakly* closed in $X^{*}$. Suppose, in addition, that the constraint qualification (14) holds at $\bar{x}$. Then there exist $\bar{\lambda}_{k}>0, \bar{\mu}_{j} \geqslant 0$ and $\bar{\nu}_{\ell} \in \mathbb{R}(k=1, \ldots, p ; j=$ 
$1, \ldots, q ; \ell=1, \ldots, r)$ such that (18) and (19) hold, in which $K$ is replaced by $T(C ; \bar{x})$.

Proof. Since $C$ is nonempty convex, $T(C ; \bar{x})$ is a nonempty closed convex cone of $X$. Applying Theorem 5.1 to $K=T(C ; \bar{x})$, we deduce the desired conclusion.

In case $X$ is finite dimensional, we obtain the following Kuhn-Tucker necessary conditions for efficiency.

Theorem 5.2. Let $\operatorname{dim} X<+\infty$, and let $\bar{x}$ be a local efficient solution to Problem (VP). Let $K$ be an arbitrary nonempty convex subcone of $T(C ; \bar{x})$ with vertex at the origin, and $K$ closed. Assume that for each $i \in\{1, \ldots, p\}$, the set $E_{i}^{*}+K^{*}$ is closed in $X^{*}$, where

$$
E_{i}=\left(\bigcap_{\substack{k=1 \\ k \neq i}}^{p} A_{k}\right) \cap\left(\bigcap_{j \in I(\bar{x})} B_{j}\right) \cap\left(\bigcap_{\ell=1}^{r} C_{\ell}\right)
$$

with $a_{k}=\nabla_{G} f_{k}(\bar{x}), b_{j}=\nabla_{G} g_{j}(\bar{x}), c_{\ell}=\nabla_{G} h_{\ell}(\bar{x})(k=1, \ldots, p ; j \in I(\bar{x}) ; \ell=$ $1, \ldots, r)$. Suppose, furthemore, that the constraint qualification (14) holds at $\bar{x}$. Then there exists $\bar{\lambda}_{k}>0, \bar{\mu}_{j} \geqslant 0$ and $\bar{\nu}_{\ell} \in \mathbb{R}(k=1, \ldots, p ; j=1, \ldots, q ; \ell=$ $1, \ldots, r)$ such that (18) and (19) hold.

Proof. Making use of Theorem 4.1, we deduce that for each $i \in\{1, \ldots, p\}$, the system (20)-(24) has no solution $v \in X$. The remainder of this proof is made in the same way as in the proof of Theorem 5.1 by using Theorem 3.2 instead of Theorem 3.1.

In case $X$ is finite dimensional and $C=X$, the following result shows that the condition of $E_{i}^{*}+T(C ; \bar{x})^{*}$ to be closed can be omitted.

Corollary 5.2. Let $\operatorname{dim} X<+\infty, C=X$, and let $\bar{x}$ be an local efficient solution of Problem (VP). Suppose that the constraint qualification (14) holds at $\bar{x}$. Then there exists $\bar{\lambda}_{k}>0, \bar{\mu}_{j} \geqslant 0$ and $\bar{\nu}_{\ell} \in \mathbb{R}(k=1, \ldots, p ; j=$ $1, \ldots, q ; \ell=1, \ldots, r)$ such that

$$
\begin{aligned}
& \sum_{k=1}^{p} \bar{\lambda}_{k} \nabla_{G} f_{k}(\bar{x})+\sum_{j=1}^{q} \bar{\mu}_{j} \nabla_{G} g_{j}(\bar{x})+\sum_{\ell=1}^{r} \bar{\nu}_{\ell} \nabla_{G} h(\bar{x})=0, \\
& \bar{\mu}_{j} g_{j}(\bar{x})=0 \quad(j=1, \ldots, q) .
\end{aligned}
$$

Proof. As also in the proof of Corollary 3.2 we have that for $C=X, T(C ; \bar{x})=$ $X$ and $E_{i}^{*}+T(C ; \bar{x})^{*}=E_{i}^{*}$. So $E_{i}^{*}+T(C ; \bar{x})^{*}$ is closed in $X^{*}$. Applying 
Theorem 5.2 to $C=X$, we deduce that there exists $\bar{\lambda}_{k}>0, \bar{\mu}_{j} \geqslant 0$ and $\bar{\nu}_{\ell} \in \mathbb{R}(k=1, \ldots, p ; j=1, \ldots, q ; \ell=1, \ldots, r)$ such that $(26)$ holds, and

$$
\sum_{k=1}^{p} \bar{\lambda}_{k}\left\langle\nabla_{G} f_{k}(\bar{x}), v\right\rangle+\sum_{j=1}^{q} \bar{\mu}_{j}\left\langle\nabla_{G} g_{j}(\bar{x}), v\right\rangle+\sum_{\ell=1}^{r} \bar{\nu}_{\ell}\left\langle\nabla_{G} h_{\ell}(\bar{x}), v\right\rangle \geqslant 0
$$

which leads to the following

$$
(\forall v \in T(C ; \bar{x})=X)
$$

$$
\sum_{k=1}^{p} \bar{\lambda}_{k} \nabla_{G} f_{k}(\bar{x})+\sum_{j=1}^{q} \bar{\mu}_{j} \nabla_{G} g_{j}(\bar{x})+\sum_{\ell=1}^{r} \bar{\nu}_{\ell} \nabla_{G} h_{\ell}(\bar{x})=0
$$

as was to be shown.

Remark 5.1. (a) From Corollary 5.2 we obtain Theorem 4.1 in [13] as a special case.

(b) If the cone $T(C ; \bar{x})$ is replaced by the cone $Z(C ; \bar{x})$, the Theorems 5.1 and 5.2 are still valid.

\section{REFERENCES}

1. S. Chandra, J. Dutta and C. S. Lalitha, Regularity conditions and optimality in vector optimization, Numer. Funct. Anal. Optim. 25 (2004), 479-501.

2. B. D. Craven and V. Jeyakumar, Equivalence of Ky Fan type minimax theorem and a Gordan type alternative theorem, Oper. Res. Letters 5 (1986), 99-102.

3. I. V. Girsanov, Lectures on Mathematical Theory of Extremum Problems, Berlin Heidenberg, Springer-Verlag, 1972.

4. G. Giorgi, B. Jiménez and V. Novo, On constraint qualifications in directionally differentiable multiobjective optimization problems, RAIRO Oper. Res. 38 (2004), 255-274.

5. I Illés and G. Kassay, Theorems of the alternative and optimality conditions for convexlike and general convexlike programming, J. Optim. Theory Appl. 101 (1999), 243-257.

6. V. Jeyakumar, Convexlike alternative theorems and mathematical programming, Optimization 16 (1985), 643-652.

7. B. Jiménez and V. Novo, Alternative theorems and necessary optimality condition for directionally differentiable multiobjective programs, J. Convex Anal. 9 (2002), 97-116.

8. B. Jiménez and V, Novo, Optimality conditions in directionally differentiable Pareto problems with a set constraint via tangent cones, Numer. Funct. Anal. Optim. 24 (2003), 557-574.

9. P. T. Kien and D, V. Luu, Optimality conditions in terms of directional derivatives, East - West J. of Mathematics 4 (2002), 119-136.

10. X. F. Li, Constraint qualifications in nonsmooth multiobjective optimization, J. Optim. Theory Appl. 106 (2000), 373-398. 
11. D. V. Luu and M. H. Nguyen, Invexity of constraint maps in mathematical programs, Nonlinear Funct. Anal. Appl. 9 (2004), 289-304.

12. D. V. Luu and P. X. Trung, Theorems of the alternative for inequality - equality systems and optimality conditions, Preprint No 16 (2004), Institute of Mathematics, Hanoi, Vietnam.

13. T. Maeda, Constraint qualifications in multiobjective optimization problems: Differentiable case, J. Optim. Theory Appl. 80 (1994), 483-500.

14. O. L. Mangasarian, Nonlinear Programming, Mc-Graw-Hill, New York, 1969.

15. V. Preda and I. Chitescu, On constraint qualification in multiobjective optimization problems: Semidifferentiable case, J. Optim. Theory Appl. 100 (1999), 417-433.

16. R. Zeng, A general Gordan alternative theorem with weakened convexity and its application, Optimization 51 (2002), 709-717.

D. V. LUU

Institute of Mathematics

18 Hoang Quoc Viet Road

10307 HANOI

VIETNAM.

M. H. NGUYEN

CERMSEM, Maison des Sciences Économiques

UNIVERsité DE PARIS 1,

106-112 BD DE L'HÔPITAL,

75647 Paris Cedex 13, France. 